\title{
Symptomatic Deterioration
}

National Cancer Institute

\section{Source}

National Cancer Institute. Symptomatic Deterioration. NCI Thesaurus. Code C124434.

A global deterioration of health status providing evidence of disease progression not covered by disease response criteria (eg. RECIST). 